\title{
Entry of Viruses into the Central Nervous System
}

\author{
JOHN K. FAZAKERLEY \\ Laboratory for Clinical and Molecular Virology University of Edinburgh, UK
}

\section{NEUROTROPIC VIRUSES}

Viral meningitis and encephalitis are not rare diseases. In adults in Europe and the USA, incidences from 11 to 26 per 100,000 population per annum have been reported for viral meningitis and 3 to 7 per 100,000 for viral encephalitis (Ponka and Pettersson, 1982; Beghi et al., 1984). Encephalitis is more common in children, a 20 year survey in Finland determined a peak incidence of 17 per 100,000 child years in the second year of life (Koskiniemi et al., 1991). In one study of an endemic area of India, the incidence of Japanese encephalitis cases was 15 per 10,000 children aged 5-9 years with total population morbidity rates estimated at 0.30 to 1.5 per 100,000 (Gajanana et al., 1995; Reuben and Gajanana, 1997). In addition to meningitis and encephalitis, viruses are associated with subacute and chronic demyelinating disease for example measles virus and subacute sclerosing panencephalitis; with dementia, for example AIDS dementia; with hydrocephalus, for example mumps virus in children; with neurodegenerative disease, for example spongiform degeneration with murine (Cas-Br-E) retroviruses; with neuro-developmental abnormalities, for example rubella virus or feline panleukopenia virus and cerebellar hypoplasia and possibly with a number of neurological diseases of unknown aetiology such as multiple sclerosis, motor neurone disease or some Parkinsonian conditions. For reviews see Johnson, 1998; Fazakerley, 2001.

Viruses which have the ability to enter the nervous system are generally designated neuroinvasive while those which have the ability to replicate there are designated neurotropic. For the purposes of this chapter

Blood-Brain Barrier

Edited by Kobiler et al., Kluwer Academic/Plenum Publishers, New York, 2001 
neuroinvasive will be used more narrowly to mean virus infection of the central nervous system. It should be noted that neurotropic denotes virus tropism for one or several types of neural cells without specifying which. The term neuronotropic or gliotropic are more specific. Some neurotropic viruses are highly specific in the cell types infected others infect several cell types. For example, rabies predominantly infects neurons, HIV microglial cells and JC virus the causative agent of progressive multifocal leukoencephalopathy, oligodendrocytes while Theiler's virus, murine picornavirus and Herpes simplex virus can infect neurons, oligodendrocytes, astrocytes and microglial cells. Experimental studies in laboratory animals have shown that neurotropic viruses are not necessarily neuroinvasive; that is there are viruses which can replicate in neural cells when experimentally inoculated directly into the brain but which do not generally gain access to the CNS when inoculated extraneurally. Neurovirulent denotes CNS disease and is frequently used to indicate that the infection is fatal. In many cases the two are linked in that virus replication and the consequent pathology are the cause of death, however care should be taken here as many viruses which enter and replicate in the CNS also infect other organ systems and neuropathology may not be the cause of death.

Viruses gain direct access to several organ systems via exposed epithelial surfaces. The skin, the gastrointestinal tract, the respiratory system and the genitourinary tract are all portals of entry for several viruses, for example papillomaviruses which cause warts, rotaviruses a common cause of infantile diarrhoea, influenza virus which causes the disease of the same name and human immunodeficiency virus, the aetiologic agent of AIDS. Following minor injury or insect bites other tissue systems such as blood and muscle can also be directly infected. Once in the blood viruses have direct access to most organ systems. Hepatitis $C$ virus and Japanese encephalitis virus enter the blood following blood transfusion or insect bites, respectively. The CNS is an exception to all this, apart from rare major injury such as head trauma, there is no direct access and the blood brain barrier excludes passive invasion by blood borne viruses.

The life cycle of viruses requires them to infect, replicate and be transmitted to new hosts. There are many viruses which have evolved to occupy an ecological niche in one or a variety of human organ systems and which are transmitted between individuals. For example, measles virus infects via the respiratory tract and thereafter replicates in a number of organ systems. Humans are the only known natural hosts for this virus and the virus is maintained only by person to person spread in sufficiently large populations. Due to the difficulty of entry and exit it seems few viruses have evolved with a requirement for replication in the CNS, rabies virus is a rare example. Why then are there so many neurological diseases caused by or associated with virus infection of the CNS? It seems likely that in most 
cases entry into and replication within the CNS are not a requirement and in many cases this property probably confers little or no selective advantage. For many viruses this phenotype is more likely to be a consequence of selective pressures dictated by replication in other tissue systems. In other words it seems likely that many viruses enter the CNS and replicate there because they can without having evolved specifically to do so.

Whereas only a few viruses have evolved to get into, to replicate in and to get out of the CNS, many viruses are able to enter into and replicate in this organ system. The lists of viruses which can enter the CNS and the list of viruses which can replicate in the CNS, in each case following either natural or experimental infection, are extensive. Viruses which have the ability to enter the CNS differ considerably in the efficiency with which they do so. Some viruses are efficiently neuroinvasive for example rabies virus which always gains access to and replicates in the CNS, other viruses are neuroinvasive only rarely. Many of these rarely or occasionally neuroinvasive viruses when they do enter the CNS can cause neurological diseases. These diseases are generally rare complications of otherwise nonneurological diseases. Examples include poliomyelitis, visna, measles inclusion body encephalitis and herpes encephalitis. Polio virus is generally an enteric infection and relative to this only rarely causes neurological disease. Experimental studies with the related Theiler's murine encephalomyelitis virus (TMEV), which is also generally an enteric infection, indicate that only 1 in every 2000 naturally infected mice develops neurological disease (Theiler and Gard, 1940). Maedi-visna virus generally causes a pneumonia in infected sheep known clinically as maedi but occasionally an inflammatory demyelinating encephalitis occurs. Measles virus infection generally results in a systemic infection characterised by skin rash but in rare cases infection results in neurologic disease, measles inclusion body encephalitis (MIBE) in young adults or subacute sclerosing panencephalitis (SSPE) in children.

While it is tempting to extrapolate and assume the incidence of clinical disease is a measure of the frequency of neuroinvasion, this seems unlikely to be the case. Determining the extent of correlation between CNS infection and clinical disease is difficult. The difficulties differ between experimental animal and human infections. Experimental model systems have problems with measuring clinical signs and symptoms. Assay of virus load in the brain following experimental extraneural inoculation indicates that it is possible for viruses to be highly efficient at neuroinvasion but rarely produce apparent clinical disease. An example would be infection of adult laboratory mice with the A7(74) strain of Semliki Forest virus. In this infection even when brain virus titres are at their highest, clinical signs are hard to detect. Another issue which is clear from experimental studies is that clinically apparent neurological disease will depend on the route of 
inoculation, the dose, the strain of the virus and the age, genetic background and immune status of the host. For humans documentation and measurement of clinical signs and symptoms is not problematic, but determining rates of CNS infection or indeed infection per se can be. In one study of Japanese encephalitis virus infection in children in an endemic area of India, the ratio of overt to inapparent infection was estimated to be 1 to 270 (Gajanana et al., 1995). It is unclear what relationship this 1 in 270 value has to the efficiency of neuroinvasion since it is not known whether neuroinvasion can occur, perhaps late in disease, without clinical manifestation. The incidence of SSPE in nonimmunised populations has been estimated to be approximately one case per million children per year (Detels, 1973) however it is possible that measles virus is more efficiently neuroinvasive than this clinical incidence suggests. In one study of 59 patients with acute uncomplicated measles, that is without any neurological signs, $30 \%$ had a pleocytosis shortly after the onset of rash, $15 \%$ had viral specific antibodies in their cerebrospinal fluid, $10 \%$ had evidence of bloodbrain barrier disturbance and $50 \%$ had abnormal electroencephalograms (Hanninen et al., 1980).

\section{HOW DO VIRUSES GAIN ACCESS TO THE CNS?}

Given that direct inoculation following for example traumatic head injury is a very rare event, how then do viruses gain access to the CNS and what determines the efficiency of neuroinvasion? There are basically two routes of entry, via the nerves and via the blood. Some viruses do both, for example Varicella zoster virus first spreads via the blood and then enters and spreads along peripheral nerves. The strain of virus can also make a difference, for example in mice reovirus type 1 spreads to the CNS via the blood whereas reovirus type 3 spreads to the CNS along nerves (Tyler et al., 1986).

\section{NERVES}

Many viruses are known to be able to track along nerves, well studied examples include polio, rabies and Herpes simplex viruses. Transmission along nerves could involve one or more of four possible pathways, transport along axons, transport in the spaces between axons, transport in the perineural lymphatics or sequential infection of supporting cells such as Schwann cells or cells of the nerve sheath. Of these, transport along axons via centripetal axonal transport mechanisms has been well established for a 
number of viruses including rabies and Herpes simplex viruses (Murphy, 1977; Kristenson et al., 1974). Passive transport in endoneural or perineural spaces or lymphatics seems less likely. As one route of drainage of the cerebrospinal fluid, neurolymph channels are contiguous with the cerebrospinal fluid, but except in rare circumstances the flow is outwards from cerebrospinal fluid to lymph so it seems unlikely that viruses would be able to enter the CNS by this route. There is no evidence supporting this route of travel. Experimental studies in mice have recorded sequential infection of Schwann cells by Herpes simplex virus but this seems to be secondary to the movement of virus along axons (Johnson 1964; Kristensson et al., 1971).

An ability to travel along nerves does not necessarily equate with an ability to enter the CNS. From the site of inoculation, usually an animal bite, rabies virus tracks along peripheral nerves both sensory and motor axons and efficiently enters the CNS. HSV also moves efficiently along peripheral axons but most studies suggest CNS infection is rare. To enter the CNS along sensory nerves a virus must generally cross at least one synapse. For some viruses this may be an insurmountable barrier. Clearly, this is not the case for rabies virus. It may not be the case for HSV either since HSV can cross at least some synapses as has been elegantly demonstrated in studies in which experimental infection with this virus has been used to delineate neuronal pathways (Ugolini et al., 1989). Other viruses which can enter the CNS along peripheral nerves include reovirus type 3, Herpes simiae, pseudorabies and Borna viruses.

\section{THE OLFACTORY NERVE}

The olfactory nerve should be considered a specialised route of nerve entry since it is the only route by which a virus can, without injury, gain direct entry from the environment into a neurone which directly transmits to the CNS. The olfactory neurones have their cell bodies in the olfactory mucosa and viruses can enter these cells from the nasal airways. The axons of these cells pass through the cribriform plate, enter the cranium and synapse with CNS neurons in the periglomerular layer of the olfactory bulb. Experimentally, several viruses including Semliki Forest virus, vesicular stomatitis virus, Herpes simplex virus and murine coronavirus have been demonstrated to enter the CNS via this route (Kaluza et al., 1987; Lunch et al., 1987; Johnson 1964; Lavi et al., 1988). Bat caves may contain high titres of rabies virus in the air and rare examples of human rabies infection as a result of visits to such caves have been recorded and are presumed to have resulted from infection via the olfactory route (Constantine, 1962). 
The frontal and temporal lobe encephalitis characteristic of Herpes simplex virus has been suggested to result from infection via the olfactory nerve (Johnson and Mims, 1968).

\section{CEREBRAL VASCULAR ENDOTHELIAL CELLS}

The second route of entry into the CNS is via the blood. Generally, the blood-brain barrier is highly effective at excluding blood-borne viruses. There are two main mechanisms of access from the blood, firstly infection of or transport across cerebral endothelial cells, meningeal cells or choroid plexus cells, secondly transport in leucocytes entering the brain. Virus replication in extraneural tissues can lead to a high titre viraemia. This is frequently seen following infection by mosquito-borne viruses. In many cases the viraemia can be in the order of 100,000,000 infectious units of virus per $\mathrm{ml}$ of blood. The titre and duration of viraemia are important determinants of neuroinvasion and neutralising antibody, cytokines, for example interferon and the efficiency of the reticuloendothelial system are important determinants of the kinetics of viraemia. For example, existing neutralising antibodies either generated by a previous infection with the same virus or cross neutralising antibodies generated by a related virus, or high levels of interferon resulting from an earlier infection can greatly suppress a viraemia. Blockade of the phagocytic potential of the reticuloendothelial system can enhance a viraemia (Mims, 1964).

Experimental studies have shown that many mosquito-borne encephalitic viruses enter the brain around cerebral blood vessels. In some cases viruses have been shown to replicate in the cerebral endothelial cells though in other cases, evidence of replication is lacking and it has been suggested that viruses are transported across these cells without replicating in them. As with most issues of virus cell tropism there are generally two categories of evidence to consider. There are studies on isolated primary cells or transformed cell lines in culture and there are studies of fresh or fixed tissue. How well tissue culture systems, especially transformed cells and single cell type cultures resemble the highly complex environment of the developing or mature nervous systems is always open to question and results from in vitro systems must always be interpreted with care.

\section{CHOROID PLEXUS}

In addition to the cerebral vascular endothelium, the choroid plexus provides a second interface between the blood and the brain. A number of 
viruses including maedi visna virus, Japanese encephalitis virus and Eastern and Western equine encephalomyelitis viruses have been demonstrated to infect choroid plexus cells (Brahic et al., 1981; Hamashima et al., 1959; Liu et al., 1970). Initiation of CNS infection by this route has been demonstrated in experimental studies with mumps virus (Wolinsky et al., 1974) and mumps virus infection of choroid plexus cells occurs in mumps meningitis in humans (Herndon et al., 1974).

\section{LEUKOCYTES}

'The CNS has no organised lymphatic system and circulating leukocytes are not observed, there is a paucity of major histocompatibility molecule expression and no antibodies or complement components are present. As a result the CNS is often referred to as an immunoprivileged site. Immunospecialised is probably more accurate as the CNS can mount florid inflammatory responses, indeed these are characteristic of viral encephalitis. During infection, major histocompatibility molecule expression is inducible, astrocytes can synthesise complement components and lymphocytes both $\mathrm{T}$ and B cells can enter the CNS. However, lymphocytes not only enter the infected CNS, activated lymphocytes have the ability to enter the CNS under normal physiological conditions (Wekerle et al., 1987; Cross et al., 1990). This constant traffic of activated lymphocytes allows the immune system to monitor the CNS for infections. Most activated lymphocytes will not encounter their specific antigen and will either exit or undergo apoptotic cell death within the CNS. Should they encounter their antigen they will trigger an inflammatory response. The number of activated lymphocytes trafficking through the CNS at any one time is very small explaining why these cells are not generally observed. A number of viruses including for example measles, mumps and HIV can infect lymphocytes. Transit within these cells, presumably as a result of immune surveillance by activated cells or alternatively following many inflammatory episodes is the mechanism by which these viruses gain access to the CNS.

\section{ALPHAVIRUSES AS AN EXAMPLE OF CNS ENTRY}

Given the difficulty of assaying the human CNS and of studying early events in the preclinical phase of natural viral infections much of our knowledge of how viruses enter the CNS, their replication once there and subsequent neuropathology has been gained from studies of experimental 
infections. Most frequently these studies have been in experimental laboratory rodents, particularly mice. Several systems have been described in detail. Among the most studied and informative has been experimental infection of laboratory mice with alphaviruses. Alphaviruses are naturally transmitted by mosquitoes and clinically produce encephalitis and arthritis in humans and other animals, notably horses. In many respects alphaviruses are illustrative of other insect borne encephalitic viruses. These include, Japanese encephalitis, California encephalitis, Louping-Ill, West Nile, Murray Valley encephalitis, St Louis encephalitis and tick-borne encephalitis viruses. Three alphaviruses have been studied in detail, Semliki Forest virus (SFV), Sindbis virus (SV) and Venezuelan Equine Encephalitis virus (VEE). SFV can enter the CNS both via the blood and along nerves and will described as a detailed example to illustrate some of the more general issues discussed above.

\section{SEMLIKI FOREST VIRUS INFECTION VIA THE BLOOD}

Extraneural experimental infection in laboratory mice mimics the natural mosquito bite. Following intraperitoneal, subcutaneous or intramuscular inoculation SFV replicates in muscles and other tissues and rapidly produces a high titre plasma viraemia. The plasma viraemia lasts for around 3 days and is brought under control by neutralising IgM antibodies. During the phase of high titre viraemia viruses cross the cerebral endothelial cells and infect adjacent cells, both neurons and oligodendrocytes resulting in striking perivascular foci of infection (Figure 1) (Fazakerley et al., 1993). Original electronmicroscopic studies of infected mouse brains did not observe virus replication in cerebral vascular endothelial cells and the authors suggested virus was transported without replicating in them (Pathak and Webb, 1974). A subsequent study of a cultured mouse endothelial (immature) cell line showed good virus replication (Dropulic and Masters, 1989). Virus replication was also observed in suckling mouse primary cerebral vascular endothelial cell cultures (Soilu-Hanninen et al., 1994). In the same study, viral RNA and proteins were detected in brain endothelial cells by in situ hybridisation and immunostaining between days 1 and 7 post-infection with the highest frequency of positive cells on days 3 and 4 . Other in situ hybridisation and immunostaining studies have not reported positive cerebral vascular endothelial cells (Fazakerley et al., 1993; Gates et al., 1984; Balluz et al., 1993).

In 3 to 4 week old mice, SFV infection of the CNS occurs by $24 \mathrm{~h}$ (Pusztai et al., 1971; Fazakerley et al., 1993) and is followed a few days 
later by a biphasic mononuclear cell pleocytosis (Parsons and Webb, 1982a). Measurement of albumin and IgG in the cerebrospinal fluid demonstrates a disturbance of the blood-brain barrier between days 4 and 10 (Parson and Webb, 1982b). Disruption of the blood-brain barrier at day 5 has also been observed by the detection of fibrinogen in the brain parenchyma adjacent to cerebral blood vessels (Soilu-Hanninen et al., 1994). The cause of the blood brain barrier disruption is unclear; it could be a direct viral effect, for example destruction of endothelial cells as is observed in culture (Soilu-Hanninen et al., 1994), a direct result of the passage of inflammatory cells across the barrier or it could be mediated by cytokines, for example interferon-gamma. After resolution of the bloodbrain barrier disturbance, immunoglobulins are synthesised locally by invading B-lymphocytes (Parsons and Webb, 1984, 1989).

Expression of cellular adhesion molecules on cerebral vascular endothelial and infiltrating mononuclear cells, cytokine expression and the phenotype of infiltrating mononuclear cells during SFV A7(74) infection have been studied in two systems BALB/c and Biozzi ABH mice (SoiluHanninen et al., 1994; 1997; Morris et al., 1997; Parsons and Webb, 1989; Smith et al., 2000). It is possible that these two mouse strains differ in the kinetics of events but for the purposes of this chapter a synthesis of events follows. By immunostaining, interleukin (IL) $-1 \alpha$ and $1 \beta$, IL-10, and TGF$\beta$ are detectable at 3 days; GMCSF, IL- 2 and TNF $\alpha$ at 7 days and IFN- $\gamma$ and IL-6 at 10 days. Increased expression of ICAM-I on cerebral vascular endothelial cells and adjacent cells occurs from day 3, with a peak somewhere between days 5 and 10. Expression of VCAM-I is maximal around 7 to 10 days and is limited to the blood vessels. Major histocompatibility type-I and -II expression are detectable on endothelial and other blood vessel associated cells by 7 days. Immunostaining studies indicate that $\mathrm{CD} 8^{+}$cells, $\mathrm{LFA}-1^{+}$cells, $\mathrm{Mac}-1^{+}$cells and VLA $-4^{+}$cells are all first observed in the infected brain at 3 days and that $\mathrm{CD} 8^{+}$cells dominate the early phase of infection while $\mathrm{CD} 4^{+} \mathrm{T}$-lymphocytes are detectable from 7 days to at least 35 days. Numbers of $\mathrm{B} 220^{+}$B-lymphocytes increase dramatically from 7 days to a peak at 21 days. Treatment of infected mice with anti-VLA-4 antibodies dramatically reduces CNS cellular infiltrates and treatment with this antibody or anti-CD8 prevents lesions of CNS demyelination which are characteristic of SFV A7(74) infection (SubakSharpe et al., 1993; Smith et al., 2000). 


\section{SEMLIKI FOREST VIRUS INFECTION VIA THE OLFACTORY NERVE}

Following intranasal infection SFV can track along the olfactory nerve to enter the CNS in the olfactory bulb (Kaluza et al., 1987; Oliver and Fazakerley, 1998). For the A7(74) strain of SFV, spread of infection along olfactory and other CNS pathways is dependant upon neuronal maturity (Oliver et al., 1997; Oliver and Fazakerley, 1998). The virus can only replicate efficiently in immature developing neurones. Following intranasal infection, in the immature brain of weanling mice virus can be observed to sequentially infect the olfactory nerve layer, periglomerular cells, mitral cells and granule cells of the olfactory bulb and then to exit into secondary olfactory areas such as the olfactory tubercle and the anterior olfactory nucleus (Fig. 1) (Oliver and Fazakerley, 1998). In the immature brain the wave of infection is followed by a wave of apoptotic cell death (Allsopp and Fazakerley, 2000; Fazakerley and Allsopp, 2001). Interestingly and in complete contrast, infection of mature neurones appears to be nondestructive and can be persistent (Fazakerley and Webb, 1987; Amor et al., 1996; Donnelly et al., 1997). In this regard, it is interesting that some, generally late forming, neuronal populations retain an immature phenotype even in the adult mouse brain and remain more susceptible to apoptosis upon virus infection. Cells of the olfactory bulb are continually renewed in adult life via the rostral migratory stream from pluripotent neural precursors in the periventricular zone (Lois and Alvarez-Buylla, 1994). It is interesting to speculate that this constant renewal is required by the apoptotic death of olfactory bulb cells following virus infection and that rapid death of these cells prevents spread of viruses into the brain along the olfactory nerve, the only direct one cell connection from the environment to the CNS. 


\section{Figure 1}
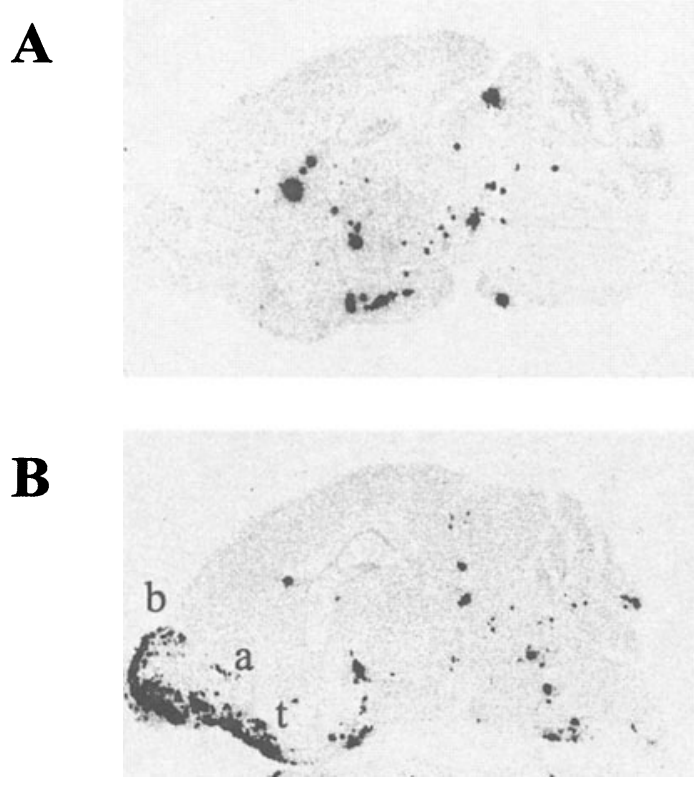

Figure 1. A. Autoradiographic image showing the distribution by in situ hybridisation of SFV A7(74) RNA in the brain following intraperitoneal infection of virus. Microscopical study of these sections shows that most of the foci of infection are centred on blood vessel. Twenty day old at infection, 72h post-infection. For details see Fazakerley et al., 1993.

B. Autoradiographic image showing the distribution by in situ hybridisation of SFV A7(74) RNA in the brain following intranasal infection of virus. Note early infection of the main olfactory bulb (b) has spread to the anterior olfactory nucleus (a) and the olfactory tubercle (t). Twenty day old at infection, 96 hours post-infection. For details see Oliver and Fazakerley, 1998. 


\section{REFERENCES}

Allsopp, T.E., and Fazakerley, J.K. 2000. Altruistic cell suicide and the specialised case of the virus-infected central nervous system. Trends in Neurosci. 23: 284-290.

Amor, S., Scallan, M.F., Morris, M.M., Dyson, H., and Fazakerley, J.K. 1996. Role of immune responses in protection and pathogenesis during Semliki Forest virus encephalitis. J. Gen. Virol. 77: 281-291.

Balluz, I.M., Glasgow, G.M., Killen, H.M., Mabruk, M.F., Sheahan, B.J., and Atkins, G.J. 1993. Virulent and avirulent strains of semliki forest virus show similar cell tropism for the murine central-nervous-system but differ in the severity and rate of induction of cytolytic damage. Neuropath. Appl. Neurobiol. 19: 233-239.

Beghi, E., Nicolosi, A., Kurland, L.T., Mulder, D.W., Hauser W.A., and Shuster, L. 1984. Encephalitis and aseptic meningitis, Olmsted County, Minnesota, 1950-1981. I. Epidemiology. Ann. Neurol. 16:283-294.

Brahic, M., Stowring, L., Ventura, P., and Haase, A.T. 1981. Gene expression in visna virus infection in sheep. Nature 292: 240.

Constantine, D.G. 1962. Rabies transmission by nonbite route. Public Health Rep. 77: 287289.

Cross, A.H., Cannella, B., Brosnan, C.F., and Raine, C.S. 1990. Homing to CNS vasculature by antigen-specific lymphocytes. Lab. Invest. 63: 162-170.

Detels, R., Brody, J.A., McNew, J., and Edgar, A.H. 1973. Further epidemiological studies of subacute sclerosing panencephalitis. Lancet 21: 11-14.

Donnelly, S.M., Sheahan, B.J., and Atkins, G.J. 1997. Long-term effects of Semliki Forest virus infection in the mouse central nervous system. Neuropath. Appl. Neurobiol. 23: 235-241.

Dropulic, B. and Masters, C.L. 1990. Entry of neurotropic arboviruses into the central nervous system: An in vitro study using mouse brain endothelium. J. Infect. Dis. 161, 685-691.

Fazakerley, J.K. 2001. Neurovirology and developmental neurobiology. Adv Vir. Res. 56: 75-126.

Fazakerley, J.K. and Webb, H.E. 1987. Semliki Forest virus-induced, immune-mediated demyelination - adoptive transfer studies and viral persistence in nude-mice. J. Gen. Virol. 68: 377-385.

Fazakerley, J.K., Pathak, S., Scallan, M., Amor, S., and Dyson, H. 1993. Replication of the A7(74) Strain of Semliki Forest virus is restricted in neurons. Virology 195: 627-637.

Fazakerley, J.K. and Allsopp, T.E. 2001. Programmed cell death in virus infections of the nervous system. Curr. Top. Microbiol. Immunol. 253: 69-93.

Gajanana, A., Thenmozhi, V., Samuel, P.P., and Reuben, R. 1995. A community-based study of subclinical flavivirus infections in children in an area of Tamil Nadu, India, where Japanese encephalitis is endemic. Bull. World Hlth Org 73: 237-44.

Gates, M.C., Sheahan, B.J., and Atkins, G.J. 1984. The pathogenicity of the M9 mutant of Semliki Forest virus in immune-compromised mice. J. Gen. Virol. 65: 73-80.

Hamashima, Y., Kyogoku, M., Hiramatusu, S., Nakashima, Y., and Yamaucki, R. 1959. Immuno-cytological studies employing labeled active protein. III. Encephalitis Japonica. Acta Pathol Jpn 9: 89-108.

Herndon, R.M., Johnson, R.T., Davis, L.E., and Descalzi, L.R. 1974. Ependymitis in mumps virus meningitis: electronmicroscopical studies of cerebrospinal fluid. Arch Neurol 30: 475-479. 
Johnson, R.T. 1964. The pathogenesis of herpes virus encephalitis. I. Virus pathways to the nervous system of suckling mice demonstrated by fluorescent antibody staining. J.Exp.Med. 119: 343-356.

Johnson, R.T. 1998. Viral infections of the nervous system (second edition), LippincottRaven; Philadelphia.

Johnson, R.T., and Mims, C.A. 1968. Medical progress, pathogenesis of viral infections of the nervous system. N. Engl. J. Med. 278: 23-30.

Kaluza, G., Lell, G., Reinacher, M., Stitz, L., and Willems, W.R. 1987. Neurogenic spread of Semliki Forest virus in mice. Arch. Virol. 93: 97-110.

Koskiniemi, M., Rautonen, J., Lehtokoski-Lehtiniemi, E., and Vaheri, A. 1991. Epidemiology of encephalitis in children: a 20-year survey. Ann. Neurol. 29: 492-497.

Kristensson, K., Lycke, E., and Sjostrand, J. 1971. Spread of herpes simplex virus in peripheral nerves. Acta Neuropathol. 17: 44-53.

Kristensson, K., Ghetti, B., and Wisniewski, H.M. 1974. Study on the propagation of herpes simplex virus (type 2) into brain after intraocular injection. Br. Res. 69: 189-201.

Lavi, E., Fishman, P.S., Highkin, M.K., and Weiss, S.R. 1988. Limbic encephalitis after inhalation of a murine coronavirus. Lab. Invest. 58: 31-36.

Liu, C., Yoth, D.W., Rodina, P., Shauf, L.R., Gonzalez, G. 1970. A comparative study of the pathogenesis of western equine and eastern equine encephalomyelitis viral infections in mice by intracerebral and subcutaneous inoculations. J. Infect Dis. 122: 53-63.

Lois, C. and Alvarez-Buylla, A. 1994. Long-distance neuronal migration in the adult mammalian brain. Science 264: 1145-1148.

Lunch, B., Kristensson, K., and Norrby, E. 1987. Selective infections of olfactory and respiratory epithelium by vesicular stomatitis and Sendai viruses. Neuropath. Appl. Neurobiol. 13: 111-122.

Mims, C.A. 1964. Aspects of the pathogenesis of virus diseases. Bacteriol. Rev. 28: 30-71.

Morris, M.M., Dyson, H., Baker, D., Harbige, L.S., Fazakerley, J.K., and Amor, S. 1997. Characterization of the cellular and cytokine response in the central nervous system following Semliki Forest virus infection. J. Neuroimmunol. 74: 185-197.

Murphy, F.A., Buchmeier, M.J., and Rawls, W.E.(1977). The reticuloendothlium as the target in a virus infection. Lab. Invest. 37: 502-515.

Oliver, K.R., Scallan, M.F., Dyson, H., and Fazakerley, J.K. 1997. Susceptibility to a neurotropic virus and its changing distribution in the developing brain is a function of CNS maturity. J. Neurovirol. 3: 38-48.

Oliver, K.R. and Fazakerley, J.K. 1998. Transneuronal spread of Semliki Forest virus in the developing mouse olfactory system is determined by neuronal maturity. Neurosci. 82: 867-877.

Parsons, L.M. and Webb, H.E. 1982. Blood brain barrier disturbance and immunoglobulin $\mathrm{G}$ levels in the cerebrospinal fluid of the mouse following peripheral infection with the demyelinating strain of Semliki Forest virus. J. Neurol. Sci. 57: 307-318.

Parsons, L.M. and Webb, H.E. 1982. Virus titers and persistently raised white cell counts in cerebrospinal-fluid in mice after peripheral infection with demyelinating Semliki Forest virus. Neuropath. Appl. Neurobiol. 8: 395-401.

Parsons, L.M. and Webb, H.E. 1984. Specific immunoglobulin G in serum and cerebrospinal fluid of mice infected with the demyelinating strain of Semliki Forest virus. Microbios Letts. 25: 135-140.

Parsons, L.M. and Webb, H.E. 1989. Identification of immunoglobulin-containing cells in the central nervous system of the mouse following infection with the demyelinating strain of Semliki Forest virus. Br. J. Exp. Pathol. 70: 247-255. 
Pathak, S. and Webb, H.E. 1974. Possible mechanisms for the transport of Semliki Forest virus into and within mouse brain: An electron microscopic study. J. Neurol. Sci. 23: 175-184.

Pathak, S. and Webb, H.E. 1980. The entry and the transport of arboviruses into and throughout mouse brain: An electron-microscopic study. Electron Microscopy 2: 492493.

Ponka, A. and Pettersson, T. 1982. The incidence and aetiology of central nervous system infections in Helsinki in 1980. Acta Neuropath. Scand. 66: 529-535.

Pusztai, R., Gould, E., and Smith, H. 1971. Infection pattern in mice of an avirulent and virulent strain of Semliki Forest virus. Br. J. Exp. Pathol. 52: 669-677.

Reuben, R., and Gajanana, A. 1997. Japanese encephalitis in India. Ind. J. Pediatr. 64: 24351.

Smith, J-P., Morris-Downes, M., Brennan, F.R., Wallace, G.J. and Amor, S. 2000. A role for $\alpha 4$-integrin in the pathology following Semliki Forest virus infection. $J$. Neuroimmunol. 106: 60-68.

SoiluHanninen, M., Eralinna, J.P., Hukkanen, V., Roytta, M., Salmi, A.A., and Salonen, R. 1994. Semliki-forest virus infects mouse-brain endothelial-cells and causes blood-brainbarrier damage. J. Virol. 68: 6291-6298.

SoiluHanninen, M., Roytta, M., Salmi, A.A., and Salonen, R. 1997. Semliki Forest virus infection leads to increased expression of adhesion molecules on splenic T-cells and on brain vascular endothelium. J. Neurovirol. 3: 350-360.

Subak-Sharpe, I., Dyson, H., and Fazakerley, J.K. 1993. In vivo depletion of CD8+ T cells prevents lesions of demyelination in Semliki Forest virus infection. J. Virol. 67: 76297633.

Theiler, M, and Gard, S. 1940. Encephalomyelitis of mice. III Epidemiology. J. Exp. Med. 79: 79-90.

Tyler, K.L., McPhee, D.A., and Fields, B.N. 1986. Distinct pathways of viral spread in the host determined by reovirus S1 gene segment. Science, 233: 770-774.

Ugolini G., Kuypers, H.G. and Strick, P.L. 1989. Transneuronal transfer of herpes virus from peripheral nerves to cortex and brainstem. Science 243: 89-91.

Wekerle, H., Sun, D., Oropeza Wekerle, R.L., and Meyermann,R. 1987. Immune reactivity in the nervous system: modulation of T-lymphocyte activation by glial cells. J. Exp. Biol. 132: 43-57.

Wolinsky, J.S., Baringer, J.R., Margolis, G., Kilham, L. 1974. Ultrastructure of mumps virus replication in newborn hamster central nervous system. Lab Invest. 31: 403-412. 